\title{
Characterization of microRNAs from goat (Capra hircus) by Solexa deep-sequencing technology
}

\author{
Y.H. Ling ${ }^{1,2 *}$, J.P. Ding ${ }^{1,2 *}$, X.D. Zhang ${ }^{1,2}$, L.J. Wang ${ }^{1,2}$, Y.H. Zhang ${ }^{1,2}$, \\ Y.S. Li ${ }^{1,2}$, Z.J. Zhang ${ }^{1,2}$ and X.R. Zhang ${ }^{1,2}$ \\ ${ }^{1}$ College of Animal Science and Technology, Anhui Agricultural University, \\ Hefei, China \\ ${ }^{2}$ Local Animal Genetic Resources Conservation and Biobreeding Laboratory \\ of Anhui Province, Hefei, China \\ *These authors contributed equally to this study. \\ Corresponding authors: X.R. Zhang / Z.J. Zhang \\ E-mail: zhangxiaorong01@163.com / zhangzijun6666@163.com
}

Genet. Mol. Res. 12 (2): 1951-1961 (2013)

Received November 29, 2012

Accepted March 30, 2013

Published June 13, 2013

DOI http://dx.doi.org/10.4238/2013.June.13.4

\begin{abstract}
MicroRNAs (miRNAs) are an important class of small noncoding RNAs that are highly conserved in plants and animals. Many miRNAs are known to mediate a myriad of cell processes, including proliferation and differentiation, via the regulation of some transcription and signaling factors, which are closely related to muscle development and disease. In this study, small RNA cDNA libraries of Boer goats were constructed. In addition, we obtained the goat muscle miRNAs by using Solexa deep-sequencing technology and analyzed these miRNA characteristics by combining it with the bioinformatics technology. Based on Solexa sequencing and bioinformatics analysis, 562 speciesconserved and 5 goat genome-specific miRNAs were identified, 322 of which exceeded 100 in the expression levels. The results of realtime quantitative polymerase chain reaction from 8 randomly selected miRNAs showed that the 8 miRNAs were expressed in goat muscle,
\end{abstract}


and the expression patterns were consistent with the Solexa sequencing results. The identification and characterization of miRNAs in goat muscle provide important information on the role of miRNA regulation in muscle growth and development. These data will help to facilitate studies on the regulatory roles played by miRNAs during goat growth and development.

Key words: Goat; Solexa deep-sequencing technology; MicroRNA characteristics; Real-time quantitative PCR

\section{INTRODUCTION}

MicroRNAs (miRNAs) are members of a class of small noncoding RNA molecules, which are critical post-transcriptional regulators of gene expression (Gunel et al., 2011). miRNAs negatively regulate gene expression through complementary base pair binding of the miRNA "seed sequence" (nucleotides 2 to 7 ) to the 3'-untranslated region of target mRNA, degrading or destabilizing the RNA message, or inhibiting protein translation, depending on the quantity of complementary base pair matches or the number of miRNA targeting sites within the 3'-untranslated region (Sandberg et al., 2008). However, studies have shown that miRNAs could increase protein translation by binding to the complementary promoter sequences (Vasudevan et al., 2007; Place et al., 2008). Numerous cellular and developmental processes in various organisms have been reported to be regulated by miRNAs, such as cell proliferation, differentiation, development, apoptosis, and disease (Chiromatzo et al., 2007; Kole et al., 2011), viral defense (Pedersen et al., 2007), hematopoietic stem cell development (Georgantas et al., 2007), environmental stress (Jones-Rhoades and Bartel, 2004), tumorigenesis (Sini et al., 2009), and the morphogenesis of specific organs (Zhao et al., 2007).

With the development of sequencing technology and bioinformatics, more and more miRNAs have been identified in animals (Ji et al., 2012). The number of miRNAs found and deposited in miRBase has risen almost exponentially. There are basically 2 kinds of approaches to identify these miRNAs. One is to sequence size-fractionated cDNA libraries. This method allows the identification of both conserved and unconserved miRNAs, but a limitation is that some miRNAs are expressed at low levels, and expressed at very specific stages or in rare cell types (Sheng et al., 2011). In contrast, the Solexa deep-sequence technology (also known as high-throughput technology) is a large massively parallel sequencing technology that utilizes sequencing by synthesis with a simply operated automatic platform. The technology has become a powerful tool for discovering miRNAs and in small RNA (sRNA) research, because of its small requirements for sample quantity and its high throughput, repeatability, and accuracy, which have led to a sharp rise in the rate of novel miRNA discoveries (Ji et al., 2012).

China has many goat breeds, including nearly 50 native goat breeds. The rearing number reached 152 million in 2010, which has been playing an important role in meat supply and agricultural improvement (Jin et al., 2012). However, there is limited information about goat miRNAs. miRNAs, which are a key component in post-transcriptional gene expression regulation in a variety of organisms, are involved in certain pathways in animal physiology and development. Several miRNAs were reported to regulate the process of skeletal muscle 
development; for example, miR-1 and -133 were found to contribute to muscular hypertrophy. We further demonstrated that miR-1 and -133 play central regulatory roles in myoblast proliferation and differentiation (Chen et al., 2010). In the present study, several miRNAs were isolated from the goat skeletal muscle, which might be researched as good candidates for taking part in the process of skeletal muscle development. Expression analysis for several miRNAs indicated that some of them were expressed in a specific tissue.

\section{MATERIAL AND METHODS}

\section{Animal and sample preparation}

Three 6-month-old Boer goats were obtained from Hefei Bo Da Livestock Technology Development Co., Ltd., which were under the same rearing condition. The animals were allowed access to feed and water ad libitum under normal conditions and were sacrificed humanely to minimize suffering. After the slaughter, the longissimus muscle tissue was surgically removed and frozen immediately in liquid nitrogen for further use.

\section{Total RNA isolation}

The longissimus muscle tissue of Boer goat was collected and used to generate sRNA libraries. Total RNA was isolated using Trizol reagent (Invitrogen, Carlsbad, CA, USA), according to manufacturer instructions. After the column purification, we used the Nanodrop ND-1000 (Nanodrop Technologies, Wilmington, DE, USA) to analyze the concentration. The quality of the total RNA was checked using the Agilent 2100 Bioanalyzer System (Agilent Technologies, Palo Alto, CA, USA) and the samples were stored at $-70^{\circ} \mathrm{C}$ until analysis.

\section{sRNA library construction and sequencing}

Total RNA was used for library preparation and sequencing by pooling equal quantities $(30 \mu \mathrm{g})$ of total RNA isolated from 3 Boer goats. Briefly, total RNA $(\sim 90 \mu \mathrm{g})$ was separated on a $10 \%$ denaturing polyacrylamide gel, and sRNAs of 18 to $26 \mathrm{nt}$ in length were recovered. Then, proprietary adapters were ligated to the $5^{\prime}$ and $3^{\prime}$ termini of the RNAs, and the samples were used as the templates for cDNA synthesis. The reverse transcription was performed, followed by PCR amplification. The PCR products were subsequently subjected to the proprietary Solexa sequencing-by-synthesis method using the Genome Analyzer (Agilent Technologies). Sequencing was carried out at the Beijing Genomics Institute, Shenzhen, China.

\section{Real-time quantitative PCR (q-PCR)}

Expressions of 8 randomly selected miRNAs identified by deep sequencing were validated by real-time q-PCR. The miRNA primers used are presented in Table 1 . The q-PCR was performed using the High-Specificity miRNA qRT-PCR Detection Kit (Qiagen, Germany) on the Roche LightCycler 480 II Real-Time PCR Detection System (LC480 II; Roche, Basel, Switzerland). The $\Delta \Delta \mathrm{Ct}$ method was used to determine the relative expression levels of miRNAs in the goat muscles. Each sample from each individual animal was analyzed 
in triplicate. Normalized factors of the internal control gene (U6 snRNA) and the relative quantities of the miRNAs were analyzed using the qBase software (Deo et al., 2011).

\begin{tabular}{llr}
\multicolumn{2}{l}{ Table 1. Primer sequences of miRNAs for q-PCR. } \\
\hline Control gene and miRNA & TaqMan probe sequences (5'-3') & Expression of Solexa sequencing \\
\hline U6 snRNA & CAAGGATGACACGCAAATTCG & - \\
miR-26a & TTCAAGTAATCCAGGATAGGCT & 75,806 \\
miR-206 & TGGAATGTAAGGAAGTGTGTGG & 614,734 \\
miR-27a & TTCACAGTGGCTAAGTTCCGC & 3,767 \\
miR-383 & AGATCAGAAGGTGATTGTGGCT & 24 \\
miR-320 & AAAAGCTGGGTTGAGAGGGCGA & 20,291 \\
miR-187 & TCGTGTCTTGTGTTGCAGCCGGA & 11 \\
miR-27b* & AGAGCTTAGCTGATTGGTGAAC & 667 \\
JR-m0005 & TAAAGTGCTTATAGTGCAGGTAG & 336 \\
\hline
\end{tabular}

\section{Data analysis}

According to the principle of bioinformatics analysis, low-quality reads were removed from the raw reads. After trimming the $3^{\prime}$ adaptor sequence, removing $5^{\prime}$ adaptor contaminants, and counting the total, unique, and length of reads, all valid sequences were obtained and used for further analysis. Sequences in the libraries with identical or related sequences to goat or other mammals were identified as conserved miRNAs. The unannotated sequences were used to predict potential novel miRNA candidates and their target genes. The SOAP software was used to analyze sRNA expression and distribution on the genome. Gene ontology analysis was performed on the predicted target gene candidates of the novel miRNAs using 3 ontologies: molecular function, cellular components, and biological process (Ji et al., 2012). All of the predicted target genes were classified according to KEGG functional annotations, to identify the pathways actively regulated by miRNAs in goat (Kanehisa et al., 2008). The databases and software used in this article are shown in Table 2.

\begin{tabular}{lll}
\multicolumn{2}{l}{ Table 2. Databases and softwares used in this article. } \\
\hline Name & Purpose & Address \\
\hline miRBase 18.0 database & Known miRNA reference sequences & $\mathrm{http}: / /$ www.mirbase.org \\
Hircine EST database & Download EST sequences & $\mathrm{http} / /$ www.ncbi.nlm.nih.gov/nucest \\
BLASTN software & Sequence alignment & $\mathrm{http}: /$ blast.ncbi.nlm.nih.gov \\
Mireap software & Novel miRNA prediction & $\mathrm{http} / /$ sourceforge.net/projects/mireap \\
SOAP software & Their expression and distribution on the genome & $\mathrm{http}: /$ soap.genomics.org.cn \\
RNAhybrid software & Target gene prediction & $\mathrm{http} / /$ bibiserv.techfak.uni-bielefeld.de/rnahybrid \\
KEGG database & Functional pathway analysis & $\mathrm{http}: / /$ www.genome.jp/kegg \\
\hline
\end{tabular}

\section{RESULTS}

\section{Extraction of goat RNA}

The sRNA was extracted with the mirVana miRNA Isolation Kit (Ambion). After removal of the small fragments of RNA, the products were detected on 1\% agarose gel electrophoresis. Compared with total RNA of ovarian tissue extracted with the Trizol method, the $5 \mathrm{~S}$ strip could barely be seen. The $18 \mathrm{~S}$ and $28 \mathrm{~S}$ strips were clear, indicating that the RNA was 
not degraded and the quality of the extracted RNA was good.

\section{Data processing and length distribution}

After gradually removing the low-quality reads and eliminating reads with 5 'primer contaminants, reads without the 3 'primer, reads without the insert tag, reads with poly(A), and reads shorter than $18 \mathrm{nt}$, the clean reads were obtained. Moreover, the sRNA species and the quantity were recorded, and then the length distribution statistics was done. In general, the interval of sRNA length is $18-30 \mathrm{nt}$, and the peak of length distribution can help us determine the sRNA type, such as miRNA focused on 21 or $22 \mathrm{nt}$, and siRNA concentrated in $24 \mathrm{nt}$ (Figure 1).

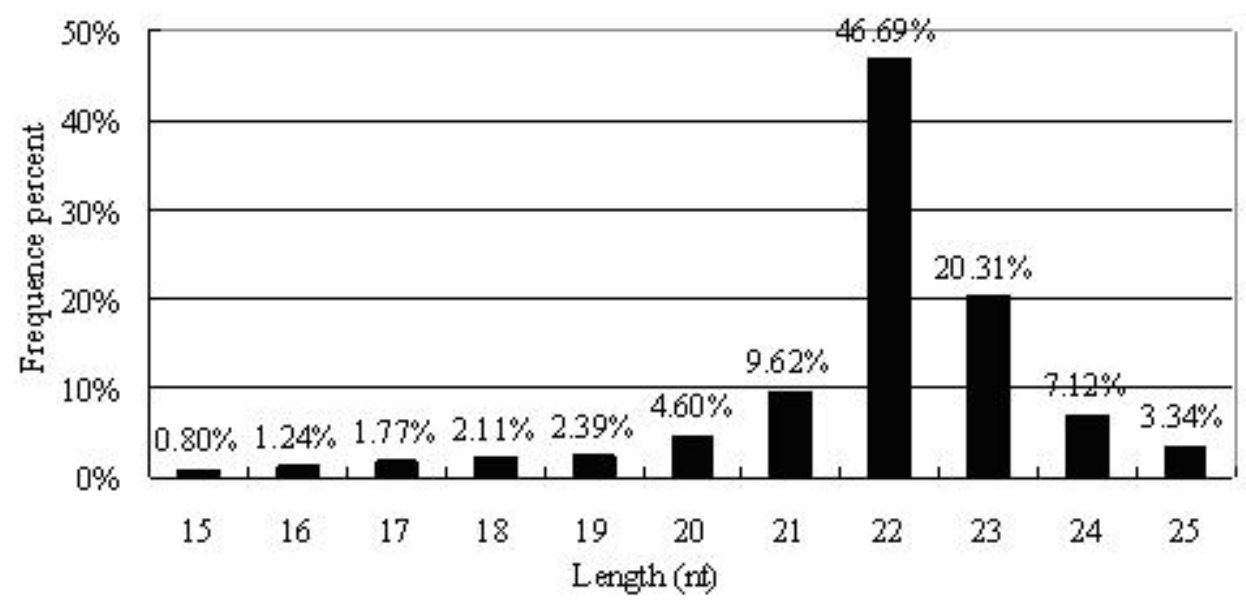

Figure 1. Length distribution of tags produced by small RNA sequencing.

\section{Genome positioning of sRNA}

The sRNA clean reads were mapped to the goat genome using the SOAP software to analyze expression and distribution of sRNAs on the genome. In this study, a total of 8,079,677 clean reads representing 402,585 unique sequences were obtained and used for further analysis. Among the sequencing data, there were 51,765 unique sRNAs matching to the genome $(12.86 \%)$, and there were 659,596 total sRNAs mapping to the genome $(8.16 \%)$ (Table 3$)$.

\section{Table 3. Mapping statistics of sample mapping to genome.}

\begin{tabular}{lcccc}
\hline & Unique sRNAs & Percent & Total sRNAs & Percent \\
\hline Total RNA & 402,585 & $100.0 \%$ & $8,079,677$ & $100.0 \%$ \\
Mapping to genome & 51,765 & $12.86 \%$ & 659,596 & $8.16 \%$ \\
\hline
\end{tabular}

\section{Expression profiling of miRNAs}

For the goat miRNAs not recorded in miRBase 18.0, all unique sequences were used 
to search the ncRNA data (GenBank, Repeat sequence, and Rfam) with BLASTN to remove non-miRNA sequences (rRNAs, tRNAs, snRNAs, snoRNA, etc.). Subsequently, the remaining sequences were analyzed using a BLAST search against miRBase 18.0. Sequences in the libraries with identical or related sequences (G-U base pairing and 1-2 nucleotide substitutions were permitted) to Ovis aries or other mammals (Bos taurus, Sus scrofa, Canis familiaris, and Equus caballus) were identified as conserved miRNAs.

There were 562 miRNAs species-conserved and 5 goat genome-specific miRNAs, 322 of which exceeded 100 in the expression levels. The expression levels of the top 20 are shown in Table 4.

\begin{tabular}{lcl}
\multicolumn{2}{c}{ Table 4. Expression level of the top 20 miRNAs in goat sample. } \\
\hline miRNA family & Expression level & \\
\hline miR-133a & $1,505,108$ & Sequence \\
miR-133c & $1,502,394$ & TTGGTCCCCTTCAACCAGCTGT \\
miR-133b & $1,426,436$ & TTGGTCCCCTCAACCAGCTG \\
miR-1 & $1,155,248$ & TTTGGTCCCCTCAACCAGCTGT \\
miR-378 & 969,945 & TGGAATGTAAAGAAGTATGTAT \\
miR-206 & 614,734 & ACTGGACTTGGAGTCAGAAGGC \\
\hline
\end{tabular}

\section{Classification and annotation of sRNA}

To further evaluate the efficiency of Solexa deep-sequencing for miRNA detection, all of the sRNA sequences were annotated and classified using the tag2 annotation software. Because some sRNA tags may be assigned to more than one category, to assign each unique sRNA to only one annotation, we followed the following priority rules: rRNA, etc. (GenBank $>$ Rfam3) $>$ known miRNA $>$ repeat $>$ exon $>$ intron (Ji et al., 2012). The results of taxonomic annotation showed that the total rRNA might be regarded as a standard: the total rRNA in better quality in animals should be less than $40 \%$ (Table 5).

\begin{tabular}{|c|c|c|c|c|}
\hline Type & Unique sRNAs & Percent & Total sRNAs & Percent \\
\hline Total & 402,585 & $100.0 \%$ & $8,079,677$ & $100.0 \%$ \\
\hline miRNA & 40,533 & $10.07 \%$ & $6,180,517$ & $76.49 \%$ \\
\hline rRNA & 48,111 & $11.95 \%$ & 762,586 & $9.44 \%$ \\
\hline repeat & 2,104 & $0.52 \%$ & 2,581 & $0.03 \%$ \\
\hline snRNA & 2,709 & $0.67 \%$ & 14,215 & $0.18 \%$ \\
\hline snoRNA & 3,082 & $0.77 \%$ & 15,332 & $0.19 \%$ \\
\hline tRNA & 9,749 & $2.42 \%$ & 74,474 & $0.92 \%$ \\
\hline unann & 296,297 & $73.60 \%$ & $1,029,972$ & $12.75 \%$ \\
\hline
\end{tabular}

\section{Prediction and expression profiling of candidate miRNAs}

For the characteristic hairpin structure of miRNA precursors that could be used to predict novel miRNAs, we developed the Mireap software (http://sourceforge.net/projects/ mireap/) to predict novel miRNAs by exploring the secondary structure, the Dicer cleavage site, and the minimum folding-free energy of the unannotated sRNA tags that could be mapped to the goat genome. Although we are currently short of substantial goat genome sequencing 
data, it is feasible to identify novel miRNAs by means of alignment with Hircine expressed sequence tags (ESTs). Therefore, we used the above software to predict the goat-specific miRNAs through extracting 50-70 nt of the ESTs (Table 6).

Table 6. Novel miRNA prediction in muscles of goat.
\begin{tabular}{lccl}
\hline Sample & Expression level & Location & Sequence \\
\hline JR-m0001 & 57 & $3^{\prime}$ & AAAATCCGAACGAACTTTTTGG \\
JR-m0002 & 576 & $5^{\prime}$ & CAAAGTGCTTACAGTGCAGGTAG \\
JR-m0003 & 28 & $5^{\prime}$ & TAAGGTGCATCTAGTGCAGA \\
JR-m0004 & 575 & $3^{\prime}$ & TGTGCAAATCTATGCAAAACTGA \\
JR-m0005 & 336 & $5^{\prime}$ & TAAAGTGCTTATAGTGCAGGTAG \\
\hline
\end{tabular}

\section{q-PCR validation of miRNAs identified in the goat muscle}

The expression levels of 8 randomly selected miRNAs were determined in the goats using q-PCR. The results from Figure 2 showed that the 8 miRNAs were expressed in the goat muscle. The expression patterns were consistent with the Solexa sequencing results (Table 1).

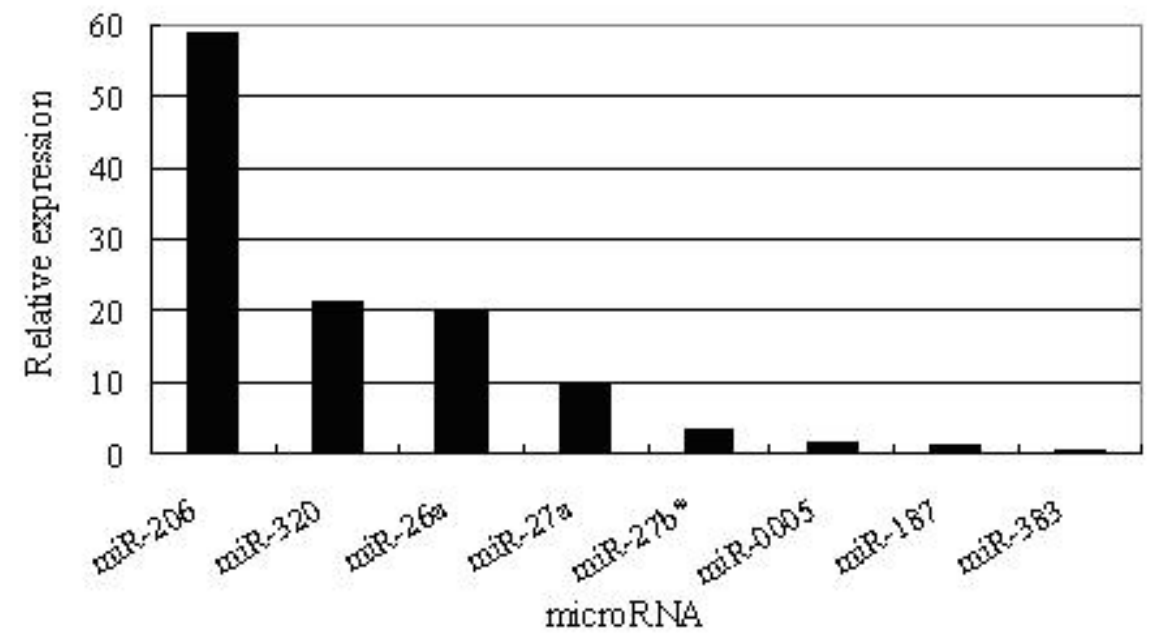

Figure 2. Relative expressions of 8 randomly selected miRNAs.

\section{miRNA target prediction and pathway analysis}

The RNAhybrid program was applied to predict the potential target sites of the identified goat miRNAs. Then, KEGG pathway analysis was also used for target gene candidates and revealed the main pathways in which the target gene candidates were involved (Allen et al., 2005; Schwab et al., 2005).

A total of 2,561,325 putative target sites for 562 miRNAs were identified in the goat muscle (data not shown). All of the predicted target genes were classified according to KEGG 
functional annotations, to identify pathways actively regulated by miRNAs in the muscle of goats. Among them, a total of 18,344 target gene sites associated with different functional pathways were identified (data not shown).

For instance, 217 target gene sites were associated with the Wnt signaling pathway, which plays an important role in many processes of life, regulating early embryonic development, cell differentiation, and cell proliferation and growth. In addition, many highly expressed miRNAs were involved in the relevant signaling pathways of growth and development. For example, the miR-133 family (miR-133a, -133b, -133c), the most highly expressed miRNA in the muscle of goat, was predicted to bind the target sequences and repress expression of the serum response factor genes. Low expression of the serum response factor genes could promote myoblast proliferation. These miRNAs were in the top 10 of expression levels, including miR-133, $-1,-378,-206$, and -143 , which play an important role in muscle growth and development.

\section{DISCUSSION}

\section{miRNAs and muscle growth and development}

Muscle growth and development result from the increase of muscle fibers and the expansion of cell volume, which will entirely depend on the muscle cell proliferation and differentiation. Some miRNAs are reported to be enriched in muscle tissues and play an important role in the regulation of myoblast proliferation and differentiation. In recent years, the genomics of relevant growth and development has gradually been elucidated from gene structure and function analyses, to determine the gene expression regulation mechanism. miRNAs perform critical roles in various biological and metabolic processes, by regulating gene expression at the post-transcriptional level.

Moreover, miRNAs are known to mediate a myriad of cell processes, including proliferation and differentiation, through the regulation of some transcription and signaling factors, which are closely related to muscle development and disease (Ge et al., 2011; Huang et al., 2012). The discovery of miRNAs points out a new path for determining the post-transcription regulatory mechanism of muscle cell proliferation and differentiation. Besides studying protein-coding genes, we also need to study the regulatory role of myogenic miRNAs, so that the molecular regulatory mechanism of skeletal muscle growth and development can be elucidated.

In this study, the goat miRNA expression profiles were systematically screened, and miRNAs were identified and characterized using Solexa deep-sequencing technology. The results indicated that sRNA libraries of the goat muscle tissue were mostly conserved miRNAs. Meanwhile, miRNAs deposited in the goat unigene database were subjected to the predicted target gene and functional analyses. We found that many highly expressed miRNAs were involved in the relevant signaling pathways of growth and development.

\section{Database selection and analysis}

Because the full goat genome has not yet been published, the complete goat miRNA information would also not be included in miRBase version 18.0 (Kozomara and Griffiths- 
Jones, 2011). The goat miRNAs were analyzed using mammalian miRNAs closely related to goat and the Hircine ESTs.

In this study, 562 species-conserved miRNAs were found, but these miRNAs were from the homologous comparison and depended on the quantity and quality of data deposited in miRBase version 18.0. Theoretically, candidate miRNAs are needed for q-PCR validation (Mestdagh et al., 2008). The expression levels of 8 randomly selected miRNAs were validated by q-PCR. The 8 miRNAs were expressed in the goat muscle, and the expression patterns were consistent with the Solexa sequencing results. These results indicated the reliability of the data.

In addition, the goat EST information was used to predict the novel miRNAs, but only 5 goat-specific miRNAs were predicted (Wei et al., 2009). Fu et al. (2011) agreed with the viewpoint that this result was caused by the scarcity of goat EST information in GenBank. If the goat genome sequence information were being constantly improved, we could take advantage of the genomic information to research the goat miRNAs, which makes the obtained results much richer and perfect.

\section{Expression profiling of muscle miRNAs}

To date, many miRNAs have been reported for their important roles in development processes in various animals (B. taurus, $S$. scrofa, and $O$. aries), but there is limited information about goat miRNAs. Sheng et al. (2011) used the cDNA library and bioinformatics methods to identify sheep miRNAs and analyzed their expression in different tissues to discover novel miRNAs. In addition, the network bioinformatics software was used to predict new sheep miRNA target genes and analyze their function. Studies indicated that a large number of miRNAs were generally coexpressed with their host genes (Drummond et al., 2011), and expression of miRNAs had tissue specificity (Zhou et al., 2010). McDaneld et al. (2009) isolated miRNAs from pig skeletal muscle tissues and found that miR-206 had a high expression in skeletal muscle. Many miRNA expression profiles have been reported in sheep, cow, chicken, and other domestic animals (Huang et al., 2010; Li et al., 2010; Wang et al., 2012), but there is limited information about goat muscle miRNAs.

In animals, miRNAs negatively regulate gene expression post-transcriptionally by translational repression and target mRNA degradation (Wienholds and Plasterk, 2005). Many miRNAs have been shown to play crucial roles in muscle development and in regulation of muscle cell proliferation and differentiation. In our study, the highly expressed miRNAs of the goat muscle may inhibit some target gene expression related to muscle cell proliferation and differentiation, thereby inhibiting the goat growth and development. In the present research, among the 10 most highly expressed miRNAs were miR-133a, $-133 c,-133 b,-1,-378,-20$, and -143 , which are common miRNAs in pigs, mice, and other animal skeletal muscle. The highest expression level of miR-1 was in the pig fattening period, and the expression level in the other periods was moderate; miR-133 in the fattening period showed moderate expression, whereas expression in the embryonic period and in newborn piglets was low.

To evaluate the roles of miRNAs in porcine skeletal muscle, miRNA expression profiles were investigated using longissimus muscle tissue from pigs at embryonic day 90 (E90) and postpartum day 120 (PD 120) (Zhou et al., 2010). During later fetal development at E90, miR-206 and let-7 were highly expressed, whereas miR-1a, -133a, -26a, and -1826 showed the highest abundance during the fast growing stage at PD 120. Studies have shown that miR-143 
can promote normal adipose cell differentiation and fat deposition (Wang et al., 2011; Yi et al., 2011). In addition, miR-143 may be involved in the mammalian gonad endocrine and in maintenance of pregnancy (Huang et al., 2012). This means that miR-143 may play an important role in mammalian development and reproduction, showing the diversity and complexity of miRNA functions, which will bring us a new challenge in the further study of miRNAs.

\section{ACKNOWLEDGMENTS}

Research supported by the National High Technology Research and Development (863) in China (\#SQ2010AA1000688008), the Natural Science Foundation of Anhui Province (\#1308085QC54), the Anhui Science and Technology Program (\#11010302108), and the China Agriculture Research System (\#11004986) funds.

\section{REFERENCES}

Allen E, Xie Z, Gustafson AM and Carrington JC (2005). microRNA-directed phasing during trans-acting siRNA biogenesis in plants. Cell 121: 207-221.

Chen JF, Tao Y, Li J, Deng Z, et al. (2010). microRNA-1 and microRNA-206 regulate skeletal muscle satellite cell proliferation and differentiation by repressing Pax7. J. Cell Biol. 190: 867-879.

Chiromatzo AO, Oliveira TY, Pereira G, Costa AY, et al. (2007). miRNApath: a database of miRNAs, target genes and metabolic pathways. Genet. Mol. Res. 6: 859-865.

Deo A, Carlsson J and Lindlof A (2011). How to choose a normalization strategy for miRNA quantitative real-time (qPCR) arrays. J. Bioinform. Comput. Biol. 9: 795-812.

Drummond MJ, McCarthy JJ, Sinha M, Spratt HM, et al. (2011). Aging and microRNA expression in human skeletal muscle: a microarray and bioinformatics analysis. Physiol. Genomics 43: 595-603.

Fu Y, Shi Z, Wu M, Zhang J, et al. (2011). Identification and differential expression of microRNAs during metamorphosis of the Japanese flounder (Paralichthys olivaceus). PLoS. One. 6: e22957.

Ge Y, Sun Y and Chen J (2011). IGF-II is regulated by microRNA-125b in skeletal myogenesis. J. Cell Biol. 192: 69-81.

Georgantas RW 3rd, Hildreth R, Morisot S, Alder J, et al. (2007). CD34+ hematopoietic stem-progenitor cell microRNA expression and function: a circuit diagram of differentiation control. Proc. Natl. Acad. Sci. U.S.A 104: 2750-2755.

Gunel T, Zeybek YG, Akcakaya P, Kalelioglu I, et al. (2011). Serum microRNA expression in pregnancies with preeclampsia. Genet. Mol. Res. 10: 4034-4040.

Huang P, Gong YZ, Peng XL and Li SJ (2010). The expression profile analysis of sexual dimorphism miRNAs expressed in chicken embryo at early stages of sex differentiation. J. Agricultura Biotechnol. 18: 1149-1155.

Huang ZP, Espinoza-Lewis R and Wang DZ (2012). Determination of miRNA targets in skeletal muscle cells. Methods Mol. Biol. 798: 475-490

Ji Z, Wang G, Xie Z, Zhang C, et al. (2012). Identification and characterization of microRNA in the dairy goat (Capra hircus) mammary gland by Solexa deep-sequencing technology. Mol. Biol. Rep. 39: 9361-9371.

Jin H, Chen HQ, Qin J, Zhu YJ, et al. (2012). The polymorphism in 5 'Regulatory region and exon 13 of PRKAG3 gene and its distribution pattern in different goat breeds. Asian J. Anim. Vet. Adv. 7: 568-577.

Jones-Rhoades MW and Bartel DP (2004). Computational identification of plant microRNAs and their targets, including a stress-induced miRNA. Mol. Cell 14: 787-799.

Kanehisa M, Araki M, Goto S, Hattori M, et al. (2008). KEGG for linking genomes to life and the environment. Nucleic Acids Res. 36: D480-D484.

Kole AJ, Swahari V, Hammond SM and Deshmukh M (2011). miR-29b is activated during neuronal maturation and targets BH3-only genes to restrict apoptosis. Genes Dev. 25: 125-130.

Kozomara A and Griffiths-Jones S (2011). miRBase: integrating microRNA annotation and deep-sequencing data. Nucleic Acids Res. 39: D152-D157.

Li HX, Wang ZY, Zhang Z, Zhou X, et al. (2010). Effects of microRNA-24 on Bovine mammary epithelial cells proliferation and apoptosis at high temperature. Sin. Agric. Sci. 43: 4732-4738.

McDaneld TG, Smith TPL, Doumit ME, Miles JR, et al. (2009). MicroRNA transcriptome profiles during swine skeletal muscle development. BMC Genomics 10: 77. 
Mestdagh P, Feys T, Bernard N, Guenther S, et al. (2008). High-throughput stem-loop RT-qPCR miRNA expression profiling using minute amounts of input RNA. Nucleic Acids Res. 36: e143.

Pedersen IM, Cheng G, Wieland S, Volinia S, et al. (2007). Interferon modulation of cellular microRNAs as an antiviral mechanism. Nature 449: 919-922.

Place RF, Li LC, Pookot D, Noonan EJ, et al. (2008). MicroRNA-373 induces expression of genes with complementary promoter sequences. Proc. Natl. Acad. Sci. U. S. A 105: 1608-1613.

Sandberg R, Neilson JR, Sarma A, Sharp PA, et al. (2008). Proliferating cells express mRNAs with shortened 3' untranslated regions and fewer microRNA target sites. Science 320: 1643-1647.

Schwab R, Palatnik JF, Riester M, Schommer C, et al. (2005). Specific effects of microRNAs on the plant transcriptome. Dev. Cell 8: 517-527.

Sheng X, Song X, Yu Y, Niu L, et al. (2011). Characterization of microRNAs from sheep (Ovis aries) using computational and experimental analyses. Mol. Biol. Rep. 38: 3161-3171.

Sini RA, Trink B and Nissan A (2009). The role of microRNA in tumorigenesis: key players or innocent bystanders. $J$. Surg. Oncol. 99: 135-136.

Townley-Tilson WH, Callis TE and Wang D (2010). MicroRNAs 1, 133, and 206: critical factors of skeletal and cardiac muscle development, function, and disease. Int. J. Biochem. Cell Biol. 42: 1252-1255.

Vasudevan S, Tong Y and Steitz JA (2007). Switching from repression to activation: microRNAs can up-regulate translation. Science 318: 1931-1934.

Wang T, Li M, Guan J, Li P, et al. (2011). MicroRNAs miR-27a and miR-143 Regulate Porcine Adipocyte Lipid Metabolism. Int. J. Mol. Sci. 12: 7950-7959.

Wang XG, Yu JF, Zhang Y, Gong DQ, et al. (2012). Identification and characterization of microRNA from chicken adipose tissue and skeletal muscle. Poult. Sci. 91: 139-149.

Wei Y, Chen S, Yang P, Ma Z, et al. (2009). Characterization and comparative profiling of the small RNA transcriptomes in two phases of locust. Genome Biol,. 10: R6.

Wienholds E and Plasterk RH (2005). MicroRNA function in animal development. FEBS Lett. 579: 5911-5922.

Yi C, Xie WD, Li F, Lv Q, et al. (2011). MiR-143 enhances adipogenic differentiation of 3T3-L1 cells through targeting the coding region of mouse pleiotrophin. FEBS Lett. 585: 3303-3309.

Zhao Y, Ransom JF, Li A, Vedantham V, et al. (2007). Dysregulation of cardiogenesis, cardiac conduction, and cell cycle in mice lacking miRNA-1-2. Cell 129: 303-317.

Zhou B, Liu HL, Shi FX and Wang JY (2010). MicroRNA expression profiles of porcine skeletal muscle. Anim. Genet. 41: 499-508. 